\title{
Conjunctival carcinoma in an immunosuppressed
} patient

We report a case of intraepithelial neoplasia associated with long-term immunosuppressive therapy in a renal allograft recipient, who was in an epidemiologically low-risk group for this tumour. Although oral and cutaneous squamous neoplasia are well recognised in immunosuppressed patients, this is the first reported case in which the ocular surface was involved. The pathogenesis is discussed.

\section{Case report}

A 33-year-old Asian woman was referred by the renal physicians to the eye department in February 1996 with a 5 month history of redness and pain in the left eye. She had received a live donor kidney transplant in 1987 and a cadaveric graft in 1992 for chronic renal failure and had been receiving cyclosporin A (CsA), oral prednisolone and azathioprine since 1987. She had previously developed an inflamed lesion on the conjunctiva which was biopsied elsewhere in 1993 and revealed carcinoma-in-situ. Unfortunately she failed to attend further appointments and could not be followed up.

On this occasion visual acuity was $6 / 6$ right eye and $6 / 9$ left eye. There was a fleshy conjunctival lesion mostly on the temporal side but straddling the peripheral cornea to almost $270^{\circ}$ in extent (Fig. 1).

Anterior chambers were quiet bilaterally and gonioscopy revealed normal angles. Examination of the posterior segment of each eye was unremarkable. She was anaemic $(\mathrm{Hb} 8.8 \mathrm{~g} / \mathrm{dl})$ with no cervical lymphadenopathy. On presentation she was taking CsA $150 \mathrm{mg}$, prednisolone $10 \mathrm{mg}$ and azathioprine $100 \mathrm{mg}$

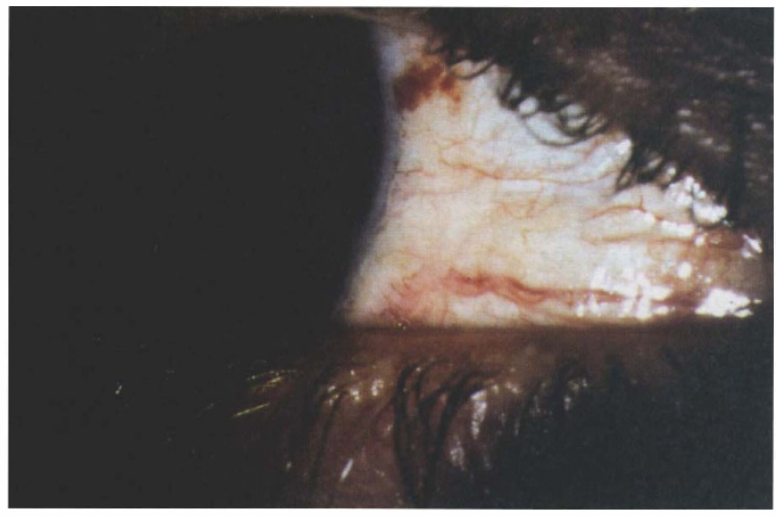

Fig. 1. Photograph of the left eye showing the conjunctival lesion adjacent to the temporal corneal limbus.

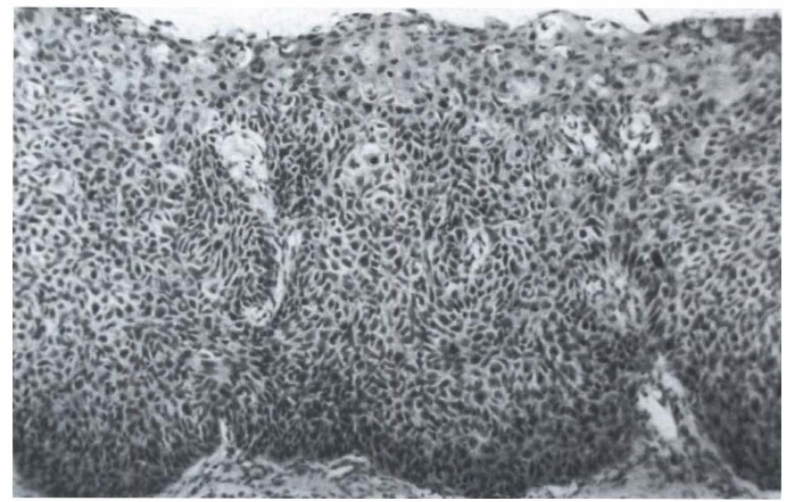

Fig. 2. Histological photomicrograph showing carcinoma-in-situ.

daily. There was no history of oral or cutaneous symptoms and the internists had not found any extraocular lesions.

Based on previous history a presumptive diagnosis of carcinoma-in-situ was made and the lesion was excised under general anaesthesia; cryotherapy was applied to the base of the excised area. Two limbal stem cell autografts were then taken from the fellow eye and sutured over the area of excision in the affected eye. A subconjunctival injection of cefuroxime $62.5 \mathrm{mg}$ and betamethasone $4 \mathrm{mg}$ was given and a bandage contact lens was placed.

Histological examination revealed full-thickness dysplasia of the conjunctival and corneal epithelia. The normal polarity and orientation of squamous cells was lost and there was marked nuclear pleomorphism and hyperchromasia. Mitotic figures were evident and scattered throughout the lesion. There was, however, no stromal invasion at any point and the basement membrane was intact (Fig. 2). These changes amounted to carcinoma-in-situ. There were no histological features of human papilloma virus (HPV) infection, including the edge of the lesion.

After 12 months of follow-up there was no evidence of disease recurrence, with a healthy ocular surface, and the patient was not taking any topical medication.

\section{Discussion}

The development of oral and cutaneous neoplastic lesions has been linked with systemic immunosuppression, but we describe the first case of ocular intraepithelial neoplasia associated with the use of systemic immunosuppression. Lee and Hirst have introduced a new terminology for neoplastic lesions of the conjunctiva and cornea. ${ }^{1}$ Ocular surface squamous neoplasia (OSSN) refers to a spectrum of disease from dysplasia to carcinoma-in-situ and squamous cell 
carcinoma involving the conjunctiva and cornea. ${ }^{1}$ It is uncommon compared with other ocular tumours, ${ }^{2}$ but in the older population it is the third most common lesion after melanoma and lymphoma. Typically OSSN occurs at the limbus in elderly Caucasian males exposed to high levels of ultraviolet B (UV-B) radiation. ${ }^{3}$ Current theories of aetiology include abnormal maturation of conjunctival and corneal epithelium secondary to factors such as UV$\mathrm{B}^{4}$ and human papilloma virus (HPV) $16 .{ }^{5}$ OSSN is usually slowly progressive and in severe untreated cases may require exenteration and can be fatal. ${ }^{4}$

The development of malignancies in immunocompromised individuals has been recognised for many years ${ }^{6}$ both in primary immunodeficiency disorders and in secondary immunodeficiency states. In the context of secondary immunodeficiency states, organ transplant recipients are particularly at risk of developing malignant tumours due to long-term immunosuppressive therapy. The commoner types of malignancy occurring in this setting are lymphomas, Kaposi's sarcoma, skin cancers, renal carcinoma, uterine cervical carcinoma, and carcinoma of the vulva and perineum. ${ }^{7}$ One study showed $7.6 \%$ of patients receiving renal allografts developed a neoplastic lesion and more than half of these were cutaneous. ${ }^{8}$ The risk of developing neoplasia increased with duration of immunosuppression.

It is well documented that the incidence of several types of malignant disease is increased in patients with AIDS. It is thought that neoplasms arising in such patients originate in the setting of decreased cellular immunity, and some of them, such as invasive cervical carcinoma, in association with HPV infection. Most of the cutaneous tumours are Kaposi's sarcoma or lymphoma. ${ }^{9}$ Other primary cutaneous malignant lesions that have been described in AIDS patients include basal cell carcinoma and squamous cell carcinoma. Indeed, HIV infection is strongly associated with an apparent increase in the incidence of conjunctival carcinoma in Africa. ${ }^{10}$

HPV has been found to play a role in inducing human malignancies. Although there were no histological features of HPV in our case, we had not performed immunohistochemistry or the polymerase chain reaction (PCR) to confirm this. HPV types 6 and 11 are associated with benign neoplasms of human squamous epithelia and types 16, 18, 31 and 33 with dysplastic lesions and carcinoma. A combination of immunohistochemistry, in situ hybridisation and PCR detected HPV infection in approximately $50 \%$ of squamous tumours of the ocular surface and lacrimal sac. ${ }^{11}$ In another study, HPV 16 and 18 were detectable with PCR not only in epithelial neoplasms of ocular mucous membranes but also in nonneoplastic lesions and healthy conjunctiva. ${ }^{12}$

In summary, epidemiological and histopathological findings strongly suggest that ultraviolet radiation is a major factor in the development of dysplastic and neoplastic changes in the conjunctiva and cornea. Another established risk factor is HIV infection acting directly or via immunosuppression, which may activate potentially oncogenic viruses such as HPV. ${ }^{13}$
Conceivably, the development of neoplasia is triggered by multiple interacting factors. ${ }^{10-12}$ In our patient, who was epidemiologically low-risk, long-term immunosuppressive therapy may well have been a factor predisposing to oncogenesis.

The number of patients undergoing immunosuppressive therapy is likely to increase in the future with the expansion of transplantation services. This will result in an increasing number of malignant conditions. This case highlights the vigilance required not only by internists but also by ophthalmologists in recognising ocular malignancies as well as previously well-documented forms of lymphopoietic and cutaneous neoplasia that can develop with the use of immunomodulation. In such patients, seemingly innocuous lesions such as pingueculae and pterygia should be followed up more closely. It is equally important to provide the patient with the necessary education to prevent late presentation of a potentially fatal condition.

\section{References}

1. Lee GA, Hirst LW. Ocular surface squamous neoplasia. Surv Ophthalmol 1995;39:429-50.

2. Templeton AC. Tumours of the eye and adnexa in Africans of Uganda. Cancer 1967;20:1689-98.

3. Ni C, Searl SS, Kriegstein HJ, Wu BF. Epibulbar carcinoma. Int Ophthalmol Clin 1982;22:1-33.

4. Tabbara KF, Kersten R, Daule N, Blodi FC. Metastatic squamous carcinoma of the conjunctiva. Ophthalmology 1988;95:318-21.

5. McDonnell JM, McDonnell PJ, Sun YY. Human papillomavirus DNA in tissues and ocular surface swabs of patients with conjunctival epithelial neoplasia. J Ophthalmol Vis Sci 1992;33:184-9.

6. Penn I. Tumour incidence in human allograft recipients. Transplant Proc 1979;11:1047-51.

7. Penn I. Immunosuppression and neoplasia. Transplant Proc 1988;20(Suppl 1):276-9.

8. London NJ, Farmery SM, Will EJ, Lodge JP. Risk of neoplasia in renal transplant patients. Lancet 1995;346:403-6.

9. Smith KJ, Skelton HG, Yeager J, Angritt P, Wagner KF. Cutaneous neoplasms in a military population of HIV-1positive patients: Military Medical Consortium for the Advancement of Retroviral Research. J Am Acad Dermatol 1993;29:400-6.

10. Waddell KM, Lewallen S, Lucas SB, Atenyi-Agaba C, Herrington CS, Liomba G. Carcinoma of the conjunctiva and HIV infection in Uganda and Malawi. Br J Ophthalmol 1996;80:503-8.

11. Nakamura Y, Mashima Y, Kamayama K, Mukai M, Oguchi Y. Detection of human papillomavirus infection in squamous tumours of the conjunctiva and lacrimal sac by immunohistochemistry, in situ hybridisation, and polymerase chain reaction. Br J Ophthalmol 1997;81:308-13.

12. Karcioglu ZA, Issa TM. Human papilloma virus in neoplastic and non-neoplastic conditions of the external eye. $\mathrm{Br} \mathrm{J}$ Ophthalmol 1997;81:595-8.

13. Newton R. A review of the aetiology of squamous cell carcinoma of the conjunctiva. Br J Cancer 1996;74:1511-3.

E.A. Ansari

K. Sahni

A.J. Morrell

Department of Ophthalmology

St James's University Hospital

Leeds LS9 7TF, UK 
N.A. Ansari

Department of Histopathology

St Thomas' Hospital

London SE 1 7EH, UK

E.A. Ansari

49 Brython Drive

St Mellons

Cardiff CF3 OLR, UK

Tel/fax: +44(0)1222798427

Sir,

Recurrent retinal artery occlusion after the disappearance of lupus anticoagulant

Antiphospholipid antibodies are a cause of recurrent arterial and venous thrombosis, and may lead to retinal vascular occlusion, especially in young people. ${ }^{1}$

Therapeutic options to prevent further vascular occlusions are varied, but it is recommended that treatment be continued until at least 6 months after the disappearance of the antibody. ${ }^{2,3}$ We describe a patient with lupus anticoagulant who had recurrent branch retinal artery occlusion (BRAO) long after the disappearance of the antibody and despite treatment with oral steroids and aspirin.

\section{Case report}

A 29-year-old women presented in November 1995, 1 week after the onset of a relative scotoma in the left eye. Visual acuity was $6 / 5$ in both eyes and a superior BRAO was found in the left eye (Fig. 1). Ocular examination was otherwise unremarkable and no disc drusen were observed on clinical examination or subsequent MRI examination of the optic nerves. Her blood pressure was $130 / 90 \mathrm{mmHg}$ and systemic examination was normal. There was no significant past ophthalmic or medical history, though she smoked 5 cigarettes daily. She had stopped taking the combined oral contraceptive pill a

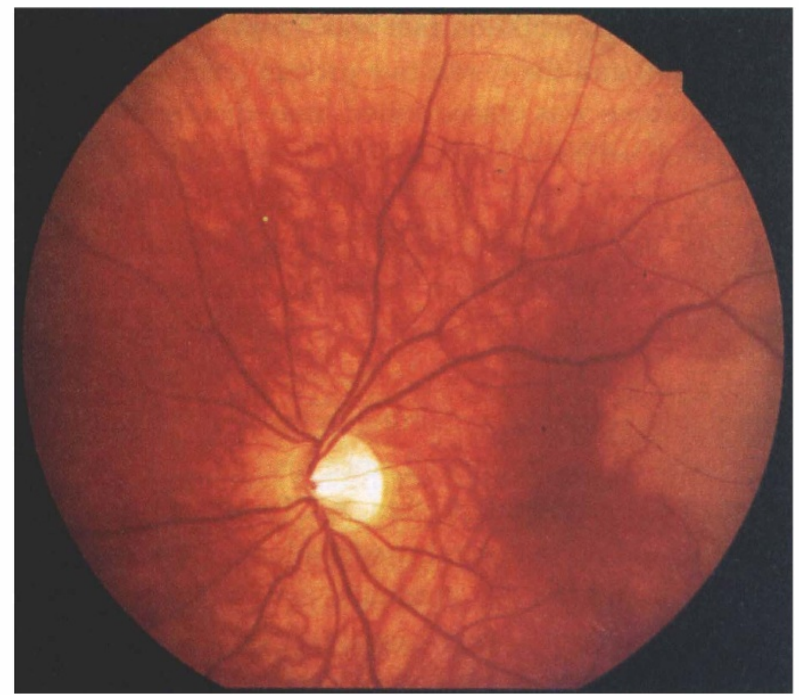

Fig. 1. Colour fundus photograph of the left eye showing an area of retinal oedema supero-temporal to the fovea that occurred secondary to a branch retinal artery occlusion. year previously, but there was no history of spontaneous abortion, migraine or intravenous drug abuse. The presence of lupus anticoagulant was confirmed that month on serological testing according to established criteria, ${ }^{5}$ but had become absent when testing was repeated in January 1996. No other hypercoagulability state was detected on either occasion. The following investigations were also negative or normal: full blood count, erythrocyte sedimentation rate, glucose,

cholesterol and an autoantibody screen including antinuclear factor and anti-neutrophil cytoplasmic antibody.

The patient presented again in April 1996 with a relative scotoma, above fixation, in the right eye. Examination revealed a supero-temporal BRAO. She was commenced on treatment with aspirin $300 \mathrm{mg}$ and prednisolone $30 \mathrm{mg}$ daily. In February 1997 a left superotemporal BRAO caused a further, relative, paracentral scotoma, although the acuity remained $6 / 5$ in both eyes. No other neurological symptoms were reported. Repeat investigation for a hypercoagulability state was unremarkable and the lupus anticoagulant was absent on each of these occasions. Echocardiography and carotid duplex imaging were unremarkable. Pure tone audiometry suggested sensorineural thresholds within normal limits. MRI examination of the brain was unremarkable and no evidence was found to suggest a more diffuse microangiopathy.

\section{Comment}

Although antiphospholipid antibodies are frequently found in patients with autoimmune and malignant disease, they are most commonly detected in patients who are otherwise healthy. ${ }^{2}$ Both lupus anticoagulant and anticardiolipin antibody may cause recurrent arterial and venous occlusion, recurrent abortion and thrombocytopenia. Retinal vascular occlusion, ischaemic optic neuropathy and amaurosis fugax have also been described. ${ }^{3,4}$ Over $50 \%$ of patients with the antiphospholipid syndrome suffer further vascular occlusion after their initial thrombotic event. ${ }^{6}$

In this patient, lupus anticoagulant was detected at the initial presentation but has been consistently undetectable since. (Serological testing was based on established criteria, ${ }^{5}$ with screening and confirmatory tests based on prolonged APTT, prolonged dRVVT and phospholipid dependency.) However, levels of lupus anticoagulant fluctuate during the course of the disease and the antibodies may disappear spontaneously, so repeated testing is necessary when the clinical suspicion is high. ${ }^{7}$ Since the initial presentation, two further arterial occlusions have occurred, 15 and 25 months after the disappearance of the antibody. Recurrent BRAO, often in young women, has previously been described by others. ${ }^{8,9}$ Although there are features in common with these previous reports, the presence of the lupus anticoagulant and the absence of symptoms or signs of vestibular or other neurological disease has led us to believe that the disease process in this patient is different. 